\title{
Modulation of oxidative phosphorylation and redox homeostasis in mitochondrial NDUFS4 deficiency via mesenchymal stem cells
}

Marlen Melcher ${ }^{1}$, Katharina Danhauser ${ }^{1}$, Annette Seibt ${ }^{1}$, Özer Degistirici ${ }^{2}$, Fabian Baertling ${ }^{1}$, Arun Kumar Kondadi ${ }^{3}$, Andreas S. Reichert ${ }^{3}$, Werner J. H. Koopman ${ }^{4}$, Peter H. G. M. Willems ${ }^{4}$, Richard J. Rodenburg ${ }^{5}$, Ertan Mayatepek', Roland Meisel ${ }^{2}$ and Felix Distelmaier ${ }^{1 *}$

\begin{abstract}
Background: Disorders of the oxidative phosphorylation (OXPHOS) system represent a large group among the inborn errors of metabolism. The most frequently observed biochemical defect is isolated deficiency of mitochondrial complex I (CI). No effective treatment strategies for Cl deficiency are so far available. The purpose of this study was to investigate whether and how mesenchymal stem cells (MSCs) are able to modulate metabolic function in fibroblast cell models of $\mathrm{Cl}$ deficiency.

Methods: We used human and murine fibroblasts with a defect in the nuclear DNA encoded NDUFS4 subunit of Cl. Fibroblasts were co-cultured with MSCs under different stress conditions and intercellular mitochondrial transfer was assessed by flow cytometry and fluorescence microscopy. Reactive oxygen species (ROS) levels were measured using MitoSOX-Red. Protein levels of Cl were analysed by blue native polyacrylamide gel electrophoresis (BN-PAGE).

Results: Direct cellular interactions and mitochondrial transfer between MSCs and human as well as mouse fibroblast cell lines were demonstrated. Mitochondrial transfer was visible in 13.2\% and 6\% of fibroblasts (e.g. fibroblasts containing MSC mitochondria) for human and mouse cell lines, respectively. The transfer rate could be further stimulated via treatment of cells with TNF-a. MSCs effectively lowered cellular ROS production in NDUFS4deficient fibroblast cell lines (either directly via co-culture or indirectly via incubation of cell lines with cell-free MSC supernatant). However, $\mathrm{Cl}$ protein expression and activity were not rescued by MSC treatment.
\end{abstract}

Conclusion: This study demonstrates the interplay between MSCs and fibroblast cell models of isolated CI deficiency including transfer of mitochondria as well as modulation of cellular ROS levels. Further exploration of these cellular interactions might help to develop MSC-based treatment strategies for human Cl deficiency.

\section{Background}

Mitochondria are important cell organelles involved in many biological processes such as aerobic metabolism of glucose and fat, calcium signalling and apoptosis regulation [1-3]. Among the metabolic pathways located within mitochondria, oxidative phosphorylation (OXPHOS) plays a prominent role in cellular energy homeostasis. The

\footnotetext{
* Correspondence: Felix.Distelmaier@med.uni-duesseldorf.de

'Department of General Pediatrics, Neonatology and Pediatric Cardiology,

University Children's Hospital, Heinrich Heine University Düsseldorf,

Moorenstraße 5, 40225 Düsseldorf, Germany

Full list of author information is available at the end of the article
}

system consists of four multi-protein complexes (CI-CIV) and the $\mathrm{F}_{0} \mathrm{~F}_{1}$-ATP synthase $(\mathrm{CV})$, embedded in the inner mitochondrial membrane $[4,5]$. Disorders of the OXPHOS system can lead to a wide range of human diseases (e.g. Leigh disease, MELAS, LHON, MERRF, etc.), frequently affecting multiple organs. They can manifest at any age, with various modes of inheritance, and the number of genetically characterized OXPHOS diseases is constantly increasing $[1,6]$. Mitochondrial CI (NADH:ubiquinone oxidoreductase) is the largest OXPHOS complex and constitutes one of the entry points for electrons into 
the electron transport chain. It consists of 44 different subunits, of which 37 are encoded by nuclear DNA (nDNA) and seven by mitochondrial DNA (mtDNA) [7, 8]. Among these subunits, the nuclear encoded NADH dehydrogenase ubiquinone Fe-S protein 4 (NDUFS4) is one of the most evolutionary conserved subunits, which is required for CI stability and function. Mutations within the NDUFS4 gene are an important cause of early-onset Leigh syndrome [9-12]. Until now, treatment options for mitochondrial diseases are mainly supportive and no curative therapeutic approach is available for affected individuals.

Bone marrow-derived mesenchymal stem cells (MSCs) were discovered in 1966 [13] and 4 years later MSCs were isolated for the first time [14]. In the following years, based on their biological properties, MSCs emerged as a promising tool for cell-based treatment strategies for various human diseases-for example autoimmune disorders [15], osteopetrosis [16] and Alzheimer's disease [17]. One prominent example for clinical application of MSCs is acute graft-versus-host disease (GVHD) during allogeneic stem cell transplantation [18]. The cellular effects of MSCs are partially related to immunosuppressive and immunomodulation effects on innate and adaptive immune cells and they escape recognition by alloreactive $\mathrm{T}$ cells $[18,19]$. MSCs have been reported to exert beneficial effects on many other cell types. Several studies demonstrated that these effects are dependent on paracrine mechanisms, including secretion of microvesicles and exosomes [20, 21]. It was shown that MSCs have the ability of intercellular organelle transport (including mitochondria) via thin (diameter 50-200 nm) cytoplasmic bridges, named tunnelling nanotubes (TNTs), or via microvesicles $[3,22$, 23]. The length of TNTs can be as long as the diameter of several cells. They contained F-actin, allowing a direct communication between distant cells. TNTs are dynamic structures that can be formed within a few minutes, but are fragile and highly sensitive to shearing forces and chemical fixatives [23, 24].

In the present study, we investigated the influence of MSCs on OXPHOS function in different cell culture systems of isolated CI deficiency caused by knockout or mutation of the NDUFS4 gene. We demonstrate that MSCs can actively transport mitochondria to fibroblast cell lines. However, in human as well as mouse fibroblast cell lines this transfer was relatively low under resting conditions. Transfer efficiency was increased moderately by stimulation of the cells with TNF- $\alpha$. The use of OPA1 knockout cells, which have a defect in mitochondrial fusion, suggested that mitochondrial transfer between MSCs and fibroblasts per individual cell is underestimated in cell lines with normal mitochondrial dynamics. We show that although mitochondrial transfer was observed, reduced CI expression and function were not rescued. However, measurements of mitochondrial reactive oxygen species (ROS) levels indicated a positive effect of mitochondrial transfer on oxidative stress. Because the use of MSC-derived cell-free culture medium also mitigated ROS production, we conclude that MSCs display effects on cellular redox homeostasis independent of mitochondrial transfer.

\section{Methods}

\section{Cells and culture conditions}

We used MSCs of the mouse strain C57BL/6 (Life Technologies). The mouse mesenchymal stem cells (mMSCs) were plated on T75 flasks (Greiner bio-one) in Dulbecco's Modified Eagle Medium/Nutrient Mixture F-12 (DMEM/F12; Gibco by life technologies) with Gluta$M^{M A X}{ }^{\text {sin }}$-I (Gibco by life technologies) supplemented with $10 \%(\mathrm{v} / \mathrm{v})$ fetal bovine serum (MSC FBS, qualified for mesenchymal stem cells; Gibco by life technologies) and $1 \%$ penicillin-streptomycin $(5000 \mathrm{U} / \mathrm{ml}$; Gibco by life technologies).

Human mesenchymal stem cells (hMSCs) were isolated as follows: 5-20 $\mathrm{ml}$ of heparinized human bone marrow aspirate was obtained from healthy volunteer donors after informed consent (this was approved by the local ethical committee of the Heinrich-Heine-University Düsseldorf; study number \#1830). The collected sample were treated with red cell lysis buffer $(155 \mathrm{mM}$ ammonium chloride, $10 \mathrm{mM}$ potassium hydrogencarbonate, $0.1 \mathrm{mM}$ Na2EDTA $\cdot 2 \mathrm{H}_{2} \mathrm{O}$; provided by the Hospital Pharmacy, University Clinic Düsseldorf, Germany) at a ratio of 1:2 and incubated for $10 \mathrm{~min}$ at room temperature. After washing with HBSS (Lonza), cells were re-suspended in complete medium consisting of Dulbecco's Modified Eagle Medium low glucose (Lonza), $2 \mathrm{mM}$ glutamin (Lonza), $1 \mathrm{IE} / \mathrm{ml} \mathrm{Na}$-heparin (Ratiopharm), 5\% human fresh or frozen plasma and $5 \%$ platelet lysate (both provided by the Institute of Hemostasis and Transfusion Medicine, University Clinic Düsseldorf, Germany), and transferred at a density of $\leq 10^{6} \mathrm{WBC} / \mathrm{cm}^{2}$ into T175 flasks (Corning). Cells were cultured at $37{ }^{\circ} \mathrm{C}$ under $10 \% \mathrm{CO}_{2}$ using a humidified incubator (Binder). Cells were allowed to adhere to the plastic surface for $24 \mathrm{~h}$ and then the medium was replaced. The removed supernatant containing nonadherent cells was transferred into new T175 flask and after further $24 \mathrm{~h}$ the supernatant with non-adherent cells was replaced by complete medium. When the cultures reached approximately $70-80 \%$ confluence, adherent cells were detached by treatment with TrypLE (Invitrogen) and re-plated into tissue flasks (all from Corning) for further passages. For experiments, hMSCs of passage 1-3 were used. The multipotentiality of the MSCs was verified with the use of invitro assays to differentiate MSCs into adipocytes (StemPro Adipogenesis Differentiation Kit; Gibco) 
and chondrocytes (type II collagen staining according to the protocol of PromoCell).

Primary human skin fibroblasts (NHDF-neo, Lot Number 7 F3956, 0000476623 and 0000478986; Lonza) and wild-type (WT) mouse fibroblasts [9] were used as control cell lines in the experiments described in the following.

Cell culture model systems of NDUFS4 deficiency included an established immortalized embryonic mouse fibroblast cell line [9], which has a knockout of NDUFS4 (NDUFS4 KO) [1], and a primary human skin fibroblast cell line from a patient with a homozygous nonsense mutation c.20C > G; p.(Ser7*) in NDUFS4 (NM_002495.2). The use of these cell lines for this study was approved by the local ethical committee of the Heinrich-HeineUniversity Düsseldorf (study number \#4272). In patient fibroblasts, NDUFS4 protein was undetectable and CI activity was drastically reduced (see Additional file 1).

For additional control experiments, we obtained immortalized embryonic mouse fibroblasts from the Institute of Biochemistry and Molecular Biology I (Medical Faculty, Heinrich-Heine-University Düsseldorf), which have a knockout of the mitochondrial inner membrane fusion protein optic atrophy 1 (OPA1 KO). All fibroblasts cell lines were cultured in DMEM with Gluta$M \mathrm{MX}^{\mathrm{m}}$-I (Gibco by life technologies) supplemented with $10 \%$ FBS and $1 \%$ penicillin-streptomycin $(5000 \mathrm{U} / \mathrm{ml})$. After fluorescence labelling via transduction, all cell lines were cultured in selection medium $(3 \mu \mathrm{g} / \mathrm{ml}$ blasticidin; Gibco by life technologies). The medium was replaced every 2-3 days, cells were split when the desired confluence $(70-90 \%)$ had been reached and the experiments started at the earliest 2 weeks after transduction.

\section{Co-culture experiments}

Fibroblasts and MSCs from the same species were cocultured in stem cell medium without blasticidin. For drug treatment experiments, cells were co-cultured on six-well plates ( $9.6 \mathrm{~cm}^{2}$ per well; Greiner CELLSTAR). For ROS measurements and fluorescence microscopy, cells were co-cultured on glass-bottom dishes $(27 \mathrm{~mm}$; Nunc by Thermo Fisher Scientific). Stem cells and fibroblasts were co-cultured in a ratio of 2:1 (drug treatment experiments) and in a ratio of 1:1 (ROS measurements and fluorescence microscopy) for $72 \mathrm{~h}$ in a humidified atmosphere (95\% air, $\left.5 \% \mathrm{CO}_{2}\right)$ at $37{ }^{\circ} \mathrm{C}$.

\section{Drug treatment}

Galactose (Gal), 2-deoxy-D-glucose (2DG), D,L-buthionine$(S, R)$-sulfoximine (BSO) and tumour necrosis factor alpha (TNF- $\alpha$ ) were obtained from Sigma-Aldrich. The fibroblasts were pre-treated for $72 \mathrm{~h}$ (Gal) or $24 \mathrm{~h}$ (BSO, 2DG). DMEM without Glucose (Gibco by life technologies) supplemented with $10 \% \mathrm{FBS}$ and $1 \%$ penicillin-streptomycin $(5,000 \mathrm{U} / \mathrm{ml})$ was used for the drug treatment experiments.
TNF- $\alpha$ was added to the cells during the co-culture. The following concentrations were used: $5.5 \mathrm{mM} \mathrm{Gal}, 20 \mathrm{mM}$ 2DG, $1 \mathrm{mM}$ and $100 \mu \mathrm{M} \mathrm{BSO}$, and 0.5, 1.0, 1.5 and $15 \mu \mathrm{g} /$ $\mathrm{ml}$ TNF- $\alpha$.

\section{SDS-PAGE and western blot analysis}

First, mitochondria were isolated as described previously [25] and the protein concentration was determined (Pierce $^{\text {Tx }}$ BCA Protein Assay Kit; ThermoFisher scientific). Then, proteins were separated by sodium dodecyl sulphate polyacrylamide gel electrophoresis (SDS-PAGE) on $4-12 \%$ protein gels (NuPAGE ${ }^{\mathrm{mm}}$ Novex $^{\mathrm{mm}} 4-12 \%$ BisTris protein gels; Invitrogen). Gels were transferred to $0.2 \mu \mathrm{m}$ nitrocellulose membrane $(1 \mathrm{~h}$ at $200 \mathrm{~mA}$; BioRad). Membranes were blocked and incubated with primary antibodies (anti-NDUFS4 (Sigma-Aldrich), polyclonal, 1:1000; voltage-dependent anion channel (VDAC; Cell Signaling), monoclonal, 1:1000; or anti-SDHA (Mitosciences MS204), 1:1000) overnight at $4{ }^{\circ} \mathrm{C}$. Horseradish peroxidase-linked anti-mouse IgG antibody (1:1000; GE Healthcare) or anti-rabbit IgG (1:1000; GE Healthcare) were used as secondary antibodies.

\section{Preparation of MSC supernatants}

The MSC supernatant preparation was performed with some modifications as described previously [21]. Briefly, medium (supernatant) from MSCs was collected and centrifuged at $300 \times g$ for $10 \mathrm{~min}$ to remove the remaining cells. Subsequently, the supernatant was transferred to a new tube and centrifuged at $2000 \times g$ for $10 \mathrm{~min}$. The cell and cell debris-free supernatant was then added to the fibroblasts. Every $24 \mathrm{~h}$, fresh cell-free MSC supernatant was added to the fibroblasts over a period of $72 \mathrm{~h}$.

\section{Fluorescent labelling}

The sequences of the organelle markers Cox8a (Cytochrome c oxidase subunit 8A) GFP, Cox8a RFP, LMNB (Lamin B1) RFP and LMNB BFP were amplified from pENTR plasmids from OriGene. A start and stop codon was added by polymerase chain reaction (PCR). Open reading frames of organelle markers were subcloned in pLenti6.3/V5-TOPO Vector using the pLenti6.3/V5TOPO TA Cloning Kit (Invitrogen). Stable cell lines from hMSCs and mMSCs with green fluorescent mitochondria (Cox8a GFP) and from human and mouse fibroblasts with red fluorescent mitochondria (Cox8a RFP) and red or blue fluorescence nucleus (LMNB RFP or LMNB BFP) were produced by lentiviral transduction. Transduction was performed according to the manufacturer's protocol with some modifications. Briefly, HEK$293 \mathrm{~T}$ cells $\left(\right.$ HEK-T) were seeded in T75 flasks $\left(1 \times 10^{6}\right.$ cells/ flask) in DMEM with 10\% FBS. After 24-48 h the HEK-T had reached a confluence of $70 \%$ and the medium was changed to $5 \mathrm{ml}$ OptiMEM (Gibco by life 
technologies). A mixture of the plasmids (pLenti6.3/V5 + insert and packing plasmids) and Lipofectamin 2000 (Invitrogen) in OptiMEM was added to the HEK-T. The medium was replaced after $24 \mathrm{~h}$ by DMEM with $10 \%$ FBS passed through a $0.45-\mu \mathrm{M}$ Millex-HV filter (Merck Millipore). Again $24 \mathrm{~h}$ later, the supernatant from the HEK-T was added to the cells to be transduced (mMSCs, MSCs, fibroblasts). Using a high-speed sorter (MoFlo XDP; Beckman-Coulter), the cells were highly enriched (RFP, GFP or BFP positive) 2 weeks after transduction.

\section{Fluorescence imaging of mitochondrial transfer}

After co-culturing, cells were stained with phalloidin (mouse cells with Alexa Fluor 594, human cells with Alexa Fluor 650 phalloidin; Molecular Probes). First, cells were fixed for $20 \mathrm{~min}$ in the dark with formalin, washed with PBS and permeabilized with $0.3 \%$ triton in PBS. Then, cells were incubated for 20 min with phalloidin. Images were obtained by fluorescence microscopy (40× objective; Zeiss; Axio Observer Z1 microscope with ApoTome 2 and a Perkin-Elmer Ultra View spinning disc confocal microscope) equipped with a GFP filterset (excitation: 520/28 BrightLine HC, emission: 482/18 BrightLine HC; AHF Analysetechnik), DAPI HC BrightLine Basic filterset BFP (excitation: 390/18 BrightLine HC, emission: 460/60 BrightLine HC; AHF Analysetechnik), Filterset 31 (excitation: BP 565/30, emission: BP 620/60; Zeiss) and Filterset 50 (excitation: BP 640/30, emission: BP 690/50; Zeiss). For live cell microscopy with recording of time-lapse videos (see Additional files 4 and 5), images were acquired at intervals of $30 \mathrm{sec}$ for a duration of $8 \mathrm{~min}$.

\section{Measurements of ROS levels}

The measurements of cellular ROS production were performed with some modifications as described previously [5]. Briefly, after co-culture for $72 \mathrm{~h}$, cells were incubated with MSC medium containing MitoSOX Red $(5 \mu \mathrm{M}$, $10 \mathrm{~min}, 37^{\circ} \mathrm{C}$; Invitrogen). The red fluorescence was documented using an Axio Observer Z1 microscope (Zeiss) with the dehydroethidium filterset (F39-500, F48515, F47-895; AHF Analysetechnik). Images were analysed and fluorescence intensity was quantified using ImageJ software (Wayne Rasband at the National Institutes of Health; http://rsbweb.nih.gov/ij/).

\section{Quantification of mitochondrial mass}

Mitochondrial mass was determined as described previously with some modifications [26]. We used Cox8alabelled fibroblasts and Cox8a-labelled MSCs for this experiment.

\section{Analysis of mitochondrial transfer by flow cytometry} After co-culture (24, 48 or $72 \mathrm{~h}$ ), cells were detached with TrypLE select (Gibco by life technologies), washed with PBS and fixed with Fixation Buffer (BioLegend) for $20 \mathrm{~min}$ in the dark. Subsequently the probes were washed again with PBS and finally re-suspended in $100 \mu \mathrm{l} \mathrm{PBS}$. A FACS Canto-II flow cytometer (BD Bioscience) was used for the measurements and data analysis was performed using FlowJo software version 10. Fibroblasts and stem cells were distinguished by their different fluorescence labelling. Only the mitochondria transfer from stem cells to fibroblasts was quantified. Approximately 30,000 cells were evaluated for each sample.

\section{Blue native polyacrylamide gel electrophoresis}

Blue native polyacrylamide gel electrophoresis (BNPAGE) was performed according to a previously described standard protocol [27] with slight modifications. In brief, mitochondria-enriched cell fractions were generated by re-suspending cell pellets in $2 \%$ digitonin followed by centrifugation. These fractions were resuspended in $2 \% n$-dodecyl $\beta$-D-maltoside to solubilize membrane proteins. Then $100 \mu \mathrm{g}$ of mitochondrial protein was loaded for each sample and separated on a 4$12 \%$ BN-PAGE gradient gel. After BN-PAGE, gels were subjected to western blotting and immunodetection as already described. Complex I in-gel-activity assay was performed by incubating the blue native gel with complex I IGA solution (3 $\mathrm{mM}$ nitrotetrazolium blue, $150 \mu \mathrm{M}$ NADH, $2 \mathrm{mM}$ Tris- $\mathrm{HCl}, \mathrm{pH}$ 7.4) for $2 \mathrm{~h}$ at room temperature immediately after the gel run. After briefly washing the gel with ultrapure water, images of the gel were captured using a gel scanner (Image Scanner III; GE Healthcare).

\section{Measurement of complex I enzyme activity}

Cells were cultured for $72 \mathrm{~h}$ with or without supernatant from MSCs. Activity of complex I was measured using a complex I enzyme activity microplate assay kit (Abcam) according to the manufacturer's protocol.

\section{Statistical analysis}

Unless otherwise described, experimental data are shown as mean \pm standard error of the mean (SEM) or standard deviation and the statistical significance between datasets is assessed using an independent two-population Student's $t$ test. $p<0.05$ was considered significant. All statistical analysis was performed using GraphPad Prism 7.

\section{Results}

\section{MSCs transfer mitochondria to fibroblasts with NDUFS4} deficiency

Mitochondrial transfer via TNTs in vitro has already been described for some cell types-for example between endothelial cells and cancer cells [28], between rat renal tubular cells and MSCs [29] and between cardiomyocytes and MSCs [22]. However, this phenomenon 
was not analysed in nuclear DNA encoded CI defects. Moreover, studies suggest that mitochondrial transfer might only be relevant in cell models with near total absence of mitochondrial function and does not play a role in human diseases caused by mitochondrial dysfunction [30].

To investigate whether MSCs are able to transfer mitochondria in two model systems of mitochondrial NDUFS4 deficiency (e.g. patient-derived fibroblasts with pathogenic NDUFS4 mutations and mouse fibroblast with NDUFS4 knockout), we performed co-culture studies of MSCs with fibroblast cell lines. For visualization,
MSC mitochondria were labelled with Cox8a GFP and fibroblast mitochondria with Cox8a RFP or the nuclei with LMNB BFP. After co-culture, cell boundaries were additionally stained with phalloidin, which binds to actin filaments. Next, cell cultures were examined by fluorescence microscopy. Video imaging studies of living cells as well as confocal microscopy studies indicated the formation of thin cytoplasmic bridges between the different cell lines, which most likely represent actin filamentbased TNTs. Moreover, for human and mouse cell lines, we observed transfer of mitochondria between MSCs and fibroblasts (Fig. 1a, b; see also Additional files 2 and

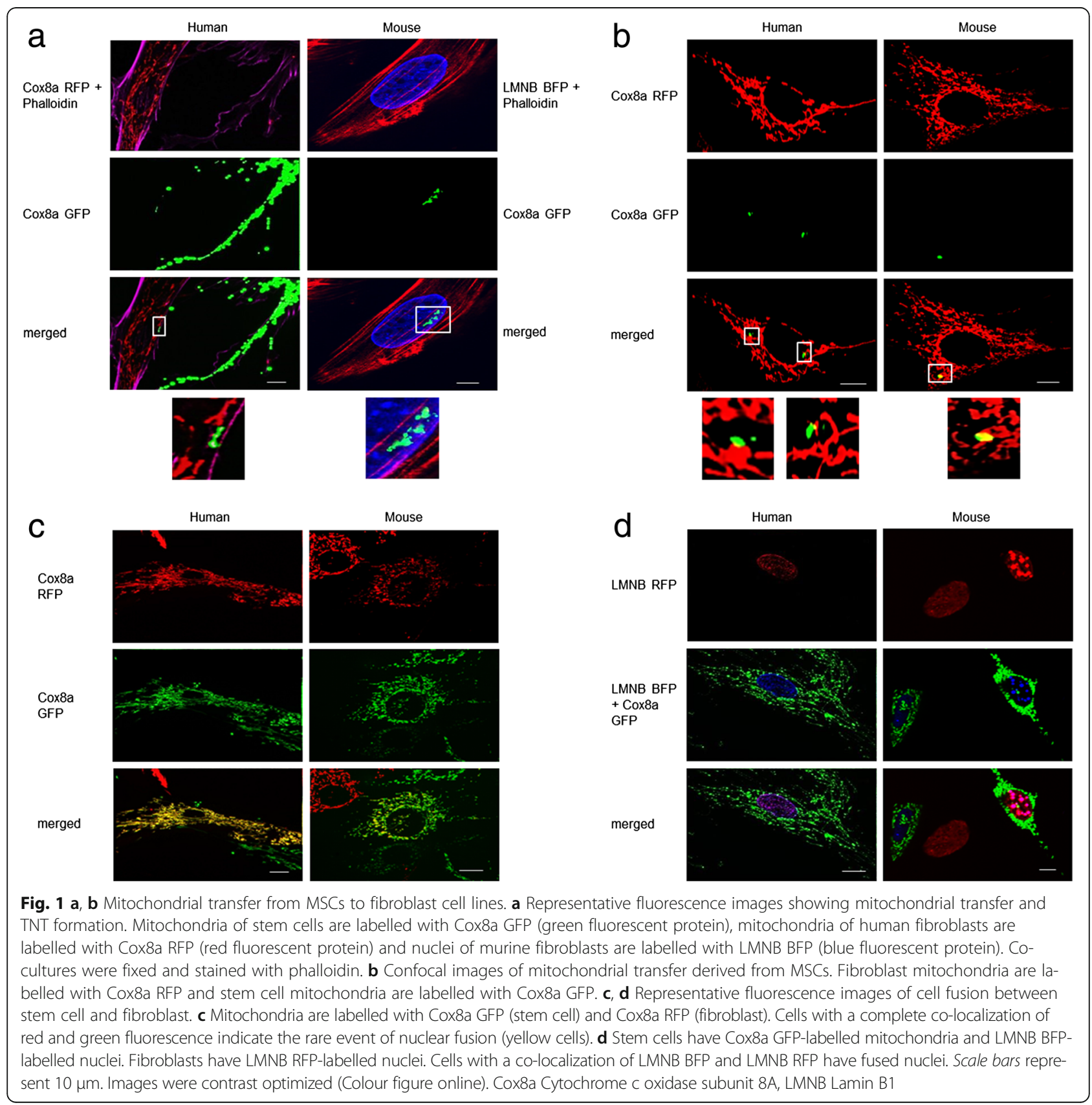


3 as well as the time-lapse videos in Additional files 4 and 5). Of note, this appeared not to be a unidirectional transfer but also occurred from fibroblasts to MSCs.

In addition to the transfer of isolated/small amounts of mitochondria we also identified cells with complete co-localization of MSC and fibroblast mitochondria (e.g. a yellow mitochondrial network; Fig. 1c). These findings suggested cellular or even nuclear fusion. To further investigate the phenomenon, we labelled MSC nuclei with LMNB BFP and mitochondria with Cox8a GFP and the fibroblast nuclei with LMNB RFP. As depicted in Fig. 1d, we observed a complete co-localization of MSC and fibroblast nuclei (Fig. 1d). This suggests complete cellular and nuclear fusion. However, at less than $0.1 \%$, the complete cell fusion appeared to be an extremely rare phenomenon (data not shown).

\section{TNF- $a$ increases the efficiency of mitochondrial transfer}

The amount of mitochondrial transfer was determined by flow cytometry using LMNB RFP-labelled fibroblasts and Cox8a GFP-labelled MSCs. After co-cultivation, a subpopulation was observed, which was LMNB RFP and Cox8a GFP positive. Next, we determined after which time period $(48,72$ or $96 \mathrm{~h}$ ) the transfer rate between patient and hMSCs or between NDUFS4 knockout and
mMSCs was most efficient (for illustration of the method used, see also Additional file 3). As depicted in Fig. 2a, transfer appeared optimal after $72 \mathrm{~h}$ (human $13.2 \%$ and mouse 6\%). For this reason, we chose for all further co-culture experiments a time period of $72 \mathrm{~h}$. Of note, we observed slight differences between the human and the murine model systems. For example, in the murine system mitochondrial transfer between NDUFS4 knockout cells and control fibroblasts was rather low (1-2\%), but in the human system the transfer between patient and control fibroblasts is almost as frequent as between patient fibroblasts and MSCs.

As shown in Fig. 2a, transfer efficiency in both model systems was relatively low. However, low transfer rate might be influenced by cell culture conditions (e.g. standard fibroblast culture provides optimal metabolic supply, which potentially masks the OXPHOS defect). Therefore, our hypothesis was that metabolic or oxidative stress might increase mitochondrial transfer.

To stimulate transfer, fibroblasts were subjected to stress condition by culturing in galactose medium as well as BSO and 2DG treatment (Fig. 2b). Galactose is a frequently used metabolic stress treatment, increasing the dependence on OXPHOS-linked ATP production [31]. BSO inhibits the glutamate cysteine ligase, which is

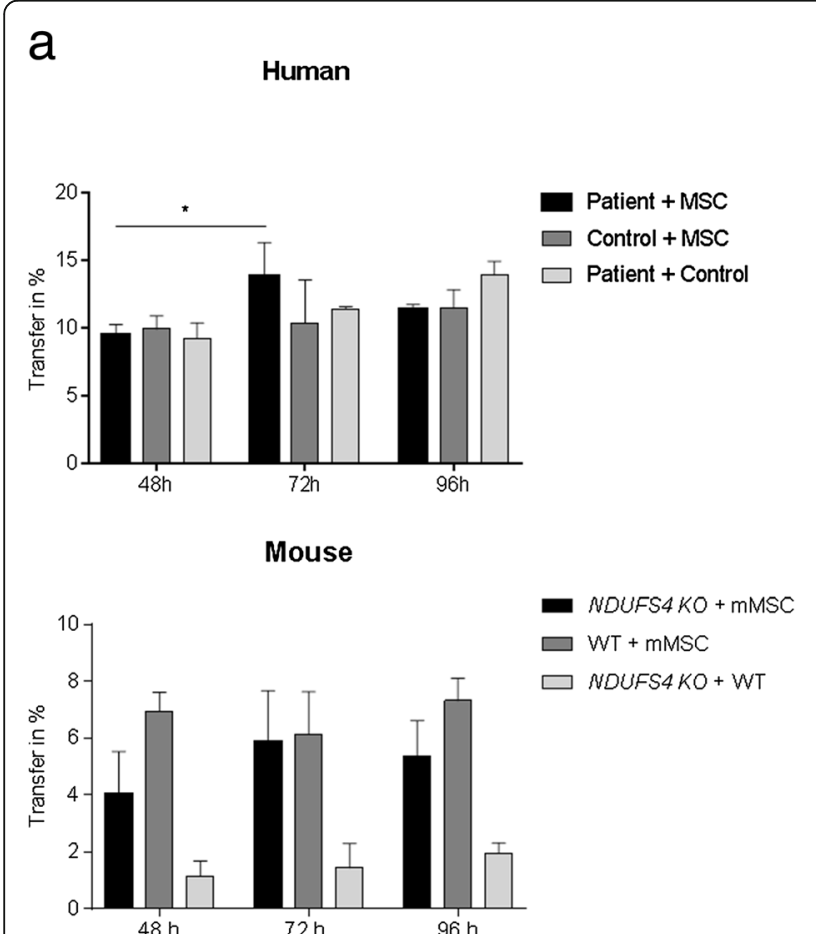

\section{b}
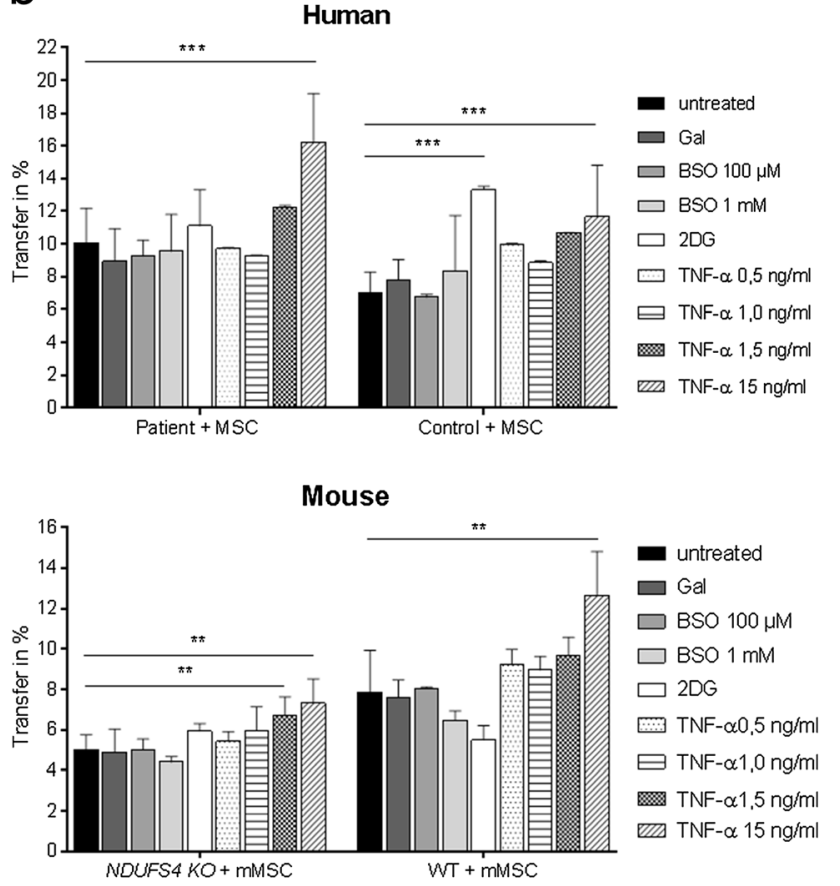

Fig. 2 Quantitative FACS measurement of mitochondrial transfer. a Flow cytometry analysis was performed after 48, 72 and $96 \mathrm{~h}$ of co-culture. Co-cultures between MSCs and fibroblasts and between fibroblasts and fibroblasts (e.g. healthy controls with NDUFS4-deficient cell lines) were measured. b Mitochondrial transfer during different culture conditions/drug treatment. mMSC mouse mesenchymal stem cell, MSC mesenchymal stem cell, Gal galactose medium, BSO buthioninesulfoximine, 2DG 2-deoxy-D-glucose, TNF-a tumour necrosis factor alpha, NDUFS4 NADH dehydrogenase ubiquinone Fe-S protein 4, WT wild type. Data shown as mean (\% of "mito-positive" cells per measurement) \pm SD. ${ }^{*} p<0.05,{ }^{* *} p<0.01,{ }^{* * *} p<0.001$ 
the rate limiting factor for glutathione synthesis. Glutathione is an important cellular antioxidant. By inhibition of the glutamate cysteine ligase, cells are exposed to oxidative stress [32]. 2DG is a glucose analogue that partially inhibits glycolysis, thereby reducing glycolytic flux. This inhibition occurs by its action on hexokinase, which is the rate limiting step of glycolysis [33].

As depicted in Fig. 2b, culturing in galactose medium and BSO treatment did not alter transfer efficiency in both model systems. Interestingly, 2DG treatment moderately increased mitochondrial transfer between human control fibroblasts and hMSCs.

Next, we evaluated the effects of TNF- $\alpha$ treatment in the different cell lines. TNF- $\alpha$ is known to stimulate the formation of TNTs by remodelling of actin filaments [34]. As shown in Fig. 2b, TNF- $\alpha$ dose-dependently stimulated transfer efficiency with significant effects at a relatively high concentration of $15 \mathrm{ng} / \mathrm{ml}$ between human patient fibroblasts and hMSCs $(10.1 \% \pm 2.1$ without TNF- $\alpha$ and $16.3 \% \pm 2.9$ with $15 \mathrm{ng} / \mathrm{ml} \mathrm{TNF-} \alpha$ ) and between NDUFS4 $\mathrm{KO}$ and mMSCs $(5 \% \pm 0.8$ without TNF- $\alpha$ and $7.4 \pm 1.2$ with $15 \mathrm{ng} / \mathrm{ml} \mathrm{TNF-} \alpha$ ).

\section{Abrogating mitochondrial fusion indicates transfer of more mitochondrial mass per individual cell}

Even during the aforementioned TNF- $\alpha$ treatment, the efficiency of mitochondrial transfer appeared to be relatively low. However, mitochondria are dynamic organelles, which constantly go through the fission/fusion cycle. Therefore, it might be possible that transferred mitochondria rapidly fuse with the mitochondrial network of the "host cell" so that the fluorescence signal is diluted and thereby the transfer rate could be underestimated. For this reason, mMSCs were co-cultured with a murine OPA1 $\mathrm{KO}$ cell line. The OPA1 protein is essential for mitochondrial fusion and cells without functional OPA1 have a fragmented mitochondrial network that is unable to incorporate/fuse mitochondria [35]. For the experiments depicted in Fig. 3a, the nuclei of the OPA1 KO fibroblasts were labelled with LMNB RFP and the mMSC mitochondria were labelled with Cox8a GFP. Transfer was analysed by flow cytometry after co-cultivation for $72 \mathrm{~h}$. A higher transfer rate between OPA1 KO fibroblast and mMSCs could not be detected (e.g. no increase in the double-stained cell population; Fig. 3a). To investigate the mitochondrial transfer on the single cell level, OPA1 KO mitochondria were labelled with Cox8a RFP and mMSC mitochondria with Cox8a GFP. Using confocal microscopy, we observed that, despite the earlier findings, larger amounts of mMSC mitochondria were transferred to OPA1 KO fibroblasts per single cell (Fig. $3 \mathrm{~b}$ ). This suggests that the images depicted in Fig. 1 potentially under-represent mitochondrial transfer because of incorporation into the mitochondrial network of the acceptor cell or because of degradation via autophagy/mitophagy. Interestingly, analysis of the mitochondrial mass in human and mouse NDUFS4-deficient fibroblasts revealed that cells with uptake of MSC mitochondria had a significant increase in mitochondrial mass (Fig. 3c). This argues against the idea that only single mitochondria are transferred and speaks against the hypothesis that transferred mitochondria are rapidly degraded via autophagy.

\section{MSCs exert antioxidative effects on NDUFS4-deficient fibroblasts}

Mitochondria are a major source of cellular ROS production via the process of OXPHOS (in particular via CI and CIII) [36]. This phenomenon might be aggravated under conditions of OXPHOS dysfunction. In fibroblasts of patients with nuclear encoded CI deficiency it was shown that superoxide production as well as hydrogen peroxide levels are significantly elevated [37].

Using the fluorescent probe MitoSOX Red, measurements of mitochondrial ROS levels were carried out via live-cell microscopy. For this analysis, stem cell mitochondria were labelled with Cox8a GFP and the nuclei of NDUFS4-deficient fibroblasts were labelled with LMNB BFP. Using this approach, we were able to measure NDUFS4-deficient fibroblasts with and without mitochondrial transfer on the same coverslip.

Results in human cell lines demonstrated increased ROS levels in NDUFS4-deficient fibroblasts (Fig. 4a, upper panel). After co-cultivation of human fibroblasts with hMSCs, the ROS levels in patient fibroblasts that had received hMSC mitochondria normalized. Figure $4 \mathrm{c}$ shows a representative fibroblast with transferred mitochondria from MSCs (green) and the MitoSOX staining (red). However, we also observed that patient fibroblasts without obvious mitochondrial transfer showed lower ROS levels compared with untreated conditions. This finding suggests that apart from direct mitochondrial transfer, additional factors (e.g. paracrine factors, microvesicles, etc.) might play a role during co-culture conditions.

Similar findings were obtained when studying the mouse cell model system (Fig. 4a, lower panel). Of note, ROS levels in mouse NDUFS4 KO cells were only elevated under low-glucose culture conditions and normalized under high-glucose conditions, which suggests differences in cell metabolism compared with primary human cells.

To investigate the effects of MSCs independent of mitochondrial transfer, we cultured fibroblasts with stem cell medium supernatant, which was free of stem cells and cell debris. To this end, every $24 \mathrm{~h}$ the supernatant was replaced by fresh supernatant and after $72 \mathrm{~h}$ we measured ROS levels using MitoSOX Red. Fibroblasts treated with stem cell supernatant produced significantly 


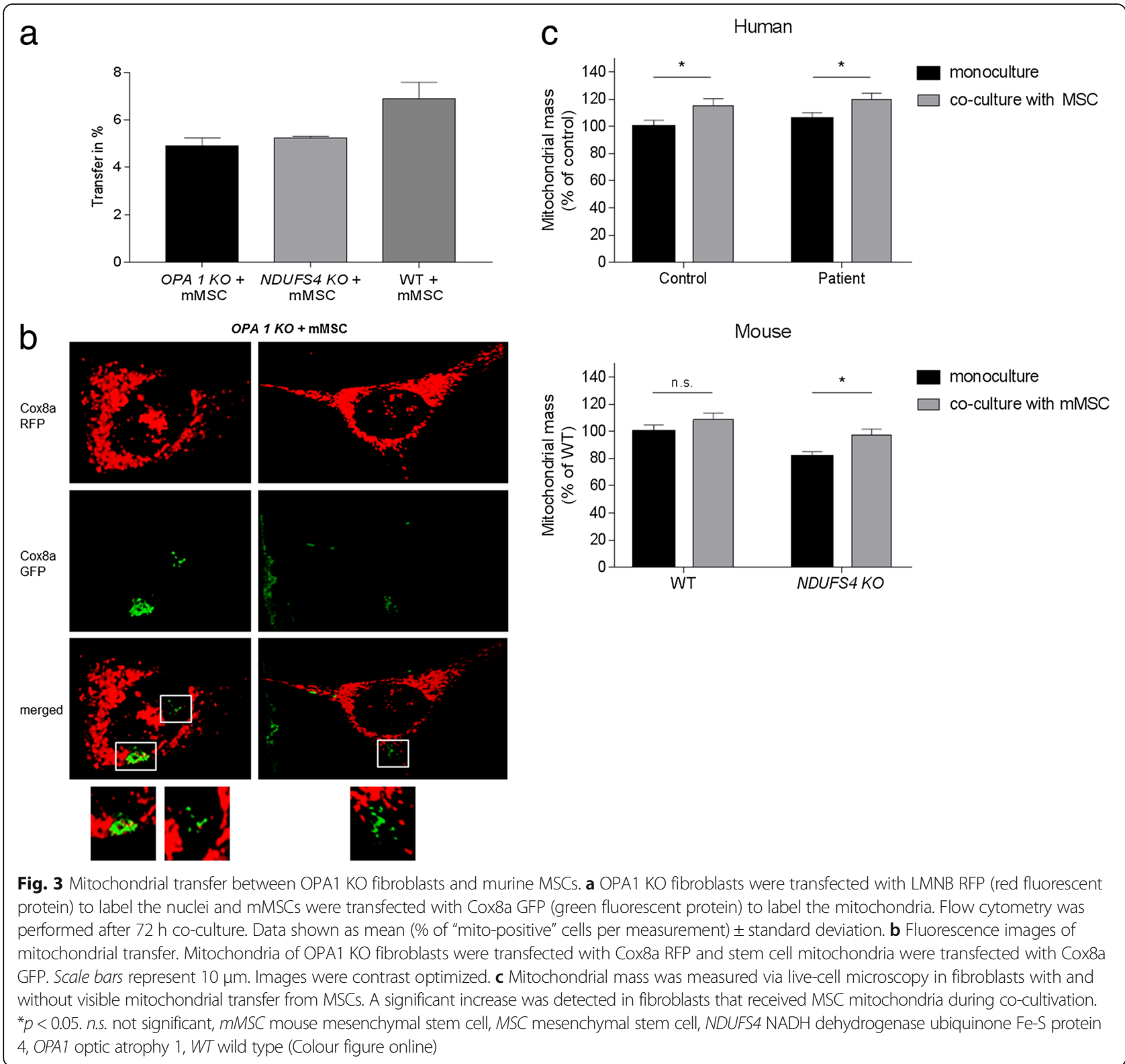

less ROS compared with untreated fibroblasts (Fig. 4b). This supports the earlier idea that MSC-derived factors are released into the cell culture medium to modulate biological functions of other cell types.

\section{Mitochondrial transfer and mitigation of cellular ROS production are unable to rescue $\mathrm{Cl}$ deficiency in NDUFS4- deficient fibroblasts}

The NDUFS4 subunit was not detectable in patient fibroblasts and in murine NDUFS4 KO fibroblasts (see Additional file $1 \mathrm{~A})$. CI activity of NDUFS4-deficient patient fibroblasts was less than $10 \%$ of the average mean in control cells. Without the NDUFS4 subunit CI is very unstable, which results in a reduced activity in NDUFS4- deficient fibroblasts [38]. To check whether treatment of fibroblasts with MSC supernatant leads to an increase of fully assembled CI, BN-PAGE was performed. Even after $72 \mathrm{~h}$ of treatment no assembled CI or CI in-gel activity could be detected in NDUFS4-deficient fibroblasts, neither in the mouse nor the human system (Fig. 5). Similar findings were obtained using a complex I enzyme activity microplate assay in mouse fibroblasts (Fig. 5c). Secreted factors of MSCs are not sufficient to increase CI levels of the NDUFS4-deficient fibroblasts to a detectable level.

\section{Discussion}

Intercellular communication is an essential process for the development and maintenance of multicellular 


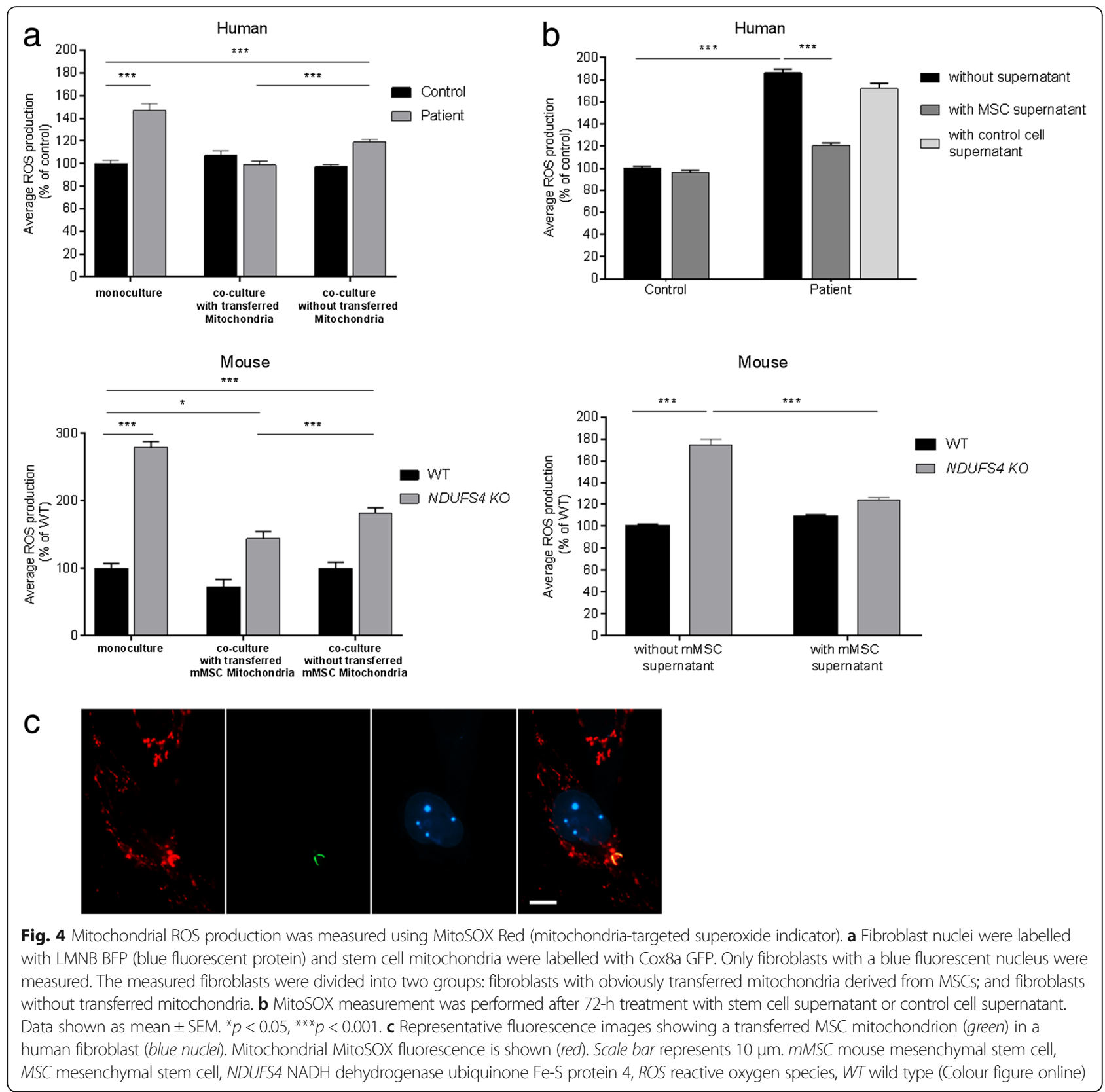

organisms. The discovery of nanotube-mediated membrane continuity between mammalian cells triggered a renewed interest in cell-cell interactions [39]. Several studies indicate that MSCs can affect the aerobic respiration of neighbouring cells by TNT-mediated mitochondrial transfer [3]. The current study aimed to determine whether mitochondrial transfer occurred from MSCs to fibroblasts with mitochondrial dysfunction due to NDUFS4 mutation (human cells) or knockout (mouse cells).

We demonstrate that, under physiological conditions, mitochondria are transferred from MSCs to fibroblasts albeit at a relatively low efficiency (6-14\%). Evidence from the literature suggested that mitochondrial transfer is increased by conditions of oxidative stress, during conditions of metabolic stress and by TNF- $\alpha$ treatment. Zhu et al. [40] analysed the effects of oxidative stress on the F-actin fibres in astrocytes. They found that increasing ROS levels via exogenous applications of $\mathrm{H}_{2} \mathrm{O}_{2}$ stimulates the polymerization of actin and thus the formation of TNT-like structures. Liu et al. [41] reported a mitochondrial transfer rate of approximately $40 \%$ between MSCs and human umbilical vein endothelial cells after oxygen glucose deprivation and subsequent reoxygenation (under normal conditions 8\%). Ahmad et al. [42] showed an increased transfer rate between mouse 
a

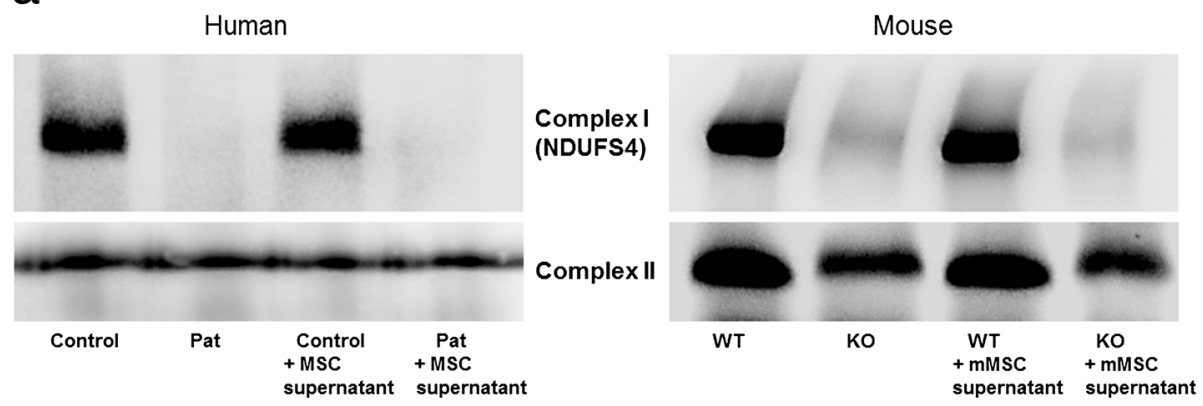

b
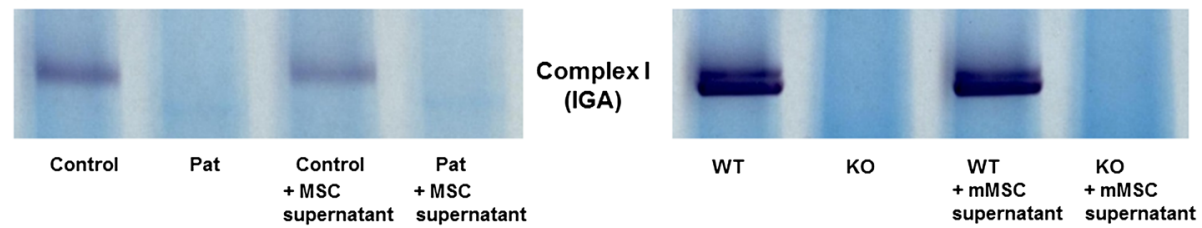

C

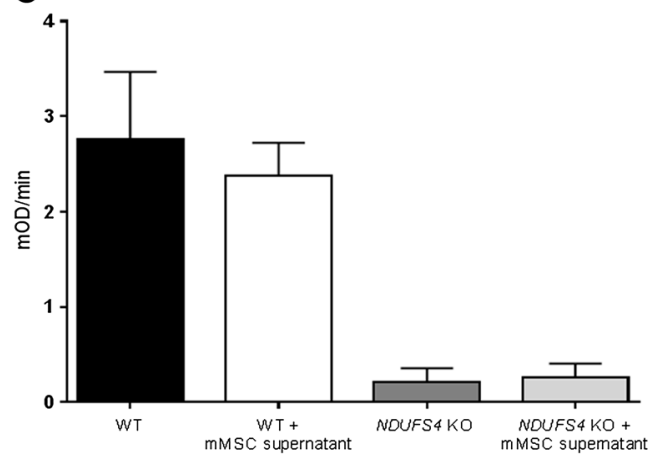

Fig. 5 a BN-PAGE with mitochondrial lysates from human and mouse fibroblasts cell lines shows no recovery of fully assembled complex I levels upon treatment with MSC supernatant. Complex II SDHA was used as loading control. b Complex I in-gel-activity assay demonstrates no improvement of CI activity upon treatment with MSC supernatant. c A complex I enzyme activity microplate assay kit was used to determine the complex I activity in mouse NDUFS4 KO and WT fibroblasts treated or untreated with supernatant from mMSCs. In accordance with BN-PAGE analysis, no significant increase in activity could be detected. Rate $(\mathrm{mOD} / \mathrm{min})=$ Absorbance 1 - Absorbance 2 / time (min). mMSC mouse mesenchymal stem cell, MSC mesenchymal stem cell, NDUFS4 NADH dehydrogenase ubiquinone Fe-S protein 4, WT wild type

lung epithelial cells (LA4) and stem cells after stress treatment with Rotenone.

Our model involving application of BSO, a substance that reduces the glutathione pool and thereby raises ROS levels, did not detectably increase mitochondrial transfer. Similarly, induction of metabolic stress by glycolysis inhibition using 2DG or GAL treatment exerted only minor (2DG) or no (GAL) effect on mitochondrial transfer. Only TNF- $\alpha$ treatment dose-dependently stimulated transfer efficiency with significant effects at a relatively high concentration. This observation suggests that MSCs respond particularly well to immunological stimuli. MSCs show immune homeostatic functions, which are enhanced by the exposure of pro-inflammatory cytokines, such as TNF- $\alpha$ [43]. High concentrations of TNF- $\alpha$ trigger the F-actin polymerization and thereby the formation of TNTs. This phenomenon most likely contributes to increased mitochondrial transfer from MSCs to fibroblasts [44].

Of note, determining mitochondrial transfer is challenging because mitochondria are constantly involved in the processes of fission and fusion. Mitochondrial fusion can be stimulated by oxidative stress and defective mitochondria may be complemented by diffusion and exchange of components between organelles [45]. Accordingly, transferred mitochondria may fuse with the network of the acceptor cell and thereby "escape" from measurements. Using an OPA1 KO cell line, which is deficient for mitochondrial fusion, we observed that individual OPA1 KO cells received 
larger amounts of mitochondria compared with what we detected in other cell lines (e.g. more transferred mitochondria per fibroblast; see Fig. 3b). However, the overall transfer rate regarding the total cell population was not increased compared with other mouse fibroblast cell lines. This suggests that mitochondrial transfer per cell might be underestimated in fibroblast lines with normal mitochondrial dynamics. In general, the fate of transferred mitochondria remains unclear. Apart from fusion with the mitochondrial network of the acceptor cell, it might also be possible that transferred mitochondria are rapidly degraded via mitophagy, which might in part be responsible for the low detection rates of "mito-positive" fibroblasts. However, the increase of mitochondrial mass in fibroblasts that received mitochondria from MSCs suggests that incorporation into the mitochondrial network of the acceptor cell is the primary mechanism.

In view of the OXPHOS defect, the main question certainly is to what extent cell contacts between MSCs and fibroblasts with mitochondrial transfer are beneficial for cell metabolism. Of note, dysfunction of the OXPHOS system is known to alter mitochondrial ROS production. As depicted in Fig. 4, levels of MitoSOX Red-oxidizing ROS were significantly increased in NDUFS4-deficient cell lines under non-treated conditions. Interestingly, in embryonic mouse fibroblasts, this increase was only detectable when culturing the cells in low-glucose medium, indicating that these cells may compensate the metabolic problem in case of high glucose supply [46]. After co-culture with MSCs, we observed a clear reduction of ROS levels in NDUFS4-deficient fibroblasts that received MSC mitochondria. In human cell lines, ROS levels even normalized completely. However, also in the "mitonegative" cell population, we observed clear effects on ROS levels indicating that the underlying mechanism might not only be related to mitochondrial transfer. This idea was further supported by the observation that treatment with cell-free MSC culture medium supernatant had similar effects on ROS levels.

MSCs are known to contain high levels of glutathione [47]. Glutathione is a substrate for glutathione peroxidase and other antioxidant enzymes, which represents a crucial cellular protection system against oxidative stress [48, 49]. Ayatollahi et al. [49] showed that oxidative stress could be significantly reduced via MSCs in rats with a carbon tetrachloride-induced liver damage. In view of the presented results, it appears highly likely that glutathione and/or other antioxidants are transferred to the NDUFS4-deficient fibroblasts (most likely via multiple mechanisms including TNTs and microvesicles), leading to the reduction in ROS levels. Modulation of cellular redox homeostasis appeared to be specific for
MSCs and was not observed after co-culture/treatment of NDUFS4-deficient fibroblasts with healthy control fibroblasts/control medium supernatant. In contrast, mitochondrial transfer was not entirely MSC specific in the human cell model system and was also visible after co-culture of NDUFS4-deficient fibroblasts with control fibroblasts.

Despite the aforementioned effects of MSCs on redox homeostasis, it remains to be answered in how far OXPHOS function can be improved in NDUFS4deficient fibroblasts via MSC treatment. However, for analysis of mitochondrial CI expression and activity, MSCs and fibroblasts need to be separated after coculture and a high purity of sorted cells is required. Moreover, relatively large amounts of cells are necessary. We were unable to achieve these experimental conditions in our co-culture system. Based on the observation that treatment of fibroblasts with MSC medium supernatant achieved similar effects on ROS levels compared with co-culture, we proceeded with analysing $\mathrm{CI}$ function in this experimental setting. As depicted in Fig. 5, treatment of NDUFS4-deficient fibroblasts with MSC supernatant had no visible effects on levels of fully assembled CI in human and mouse cell lines.

\section{Conclusions}

This study clearly indicates cellular interactions and mitochondrial transfer between MSCs and human as well as mouse fibroblast cell lines. However, mitochondrial transfer appeared to be a relatively rare phenomenon and the transfer rate could only be stimulated moderately by TNF- $\alpha$ treatment. The fate and functional relevance of transferred mitochondria remained elusive. Moreover, mitochondrial transfer in our cell culture systems was not entirely specific for MSCs and was also observed between control and patient-derived fibroblasts. In contrast, MSCs effectively lowered cellular ROS levels in NDUFS4-deficient fibroblast cell lines. This appeared to be a phenomenon that was at least partially independent of mitochondrial transfer and is putatively mediated via secreted factors (e.g. microvesicles). Modulation of cellular redox homeostasis was MSC specific and could not be achieved via control fibroblast cell lines. However, CI expression and activity could not be rescued by treatment with MSC medium supernatant. In view of this observation and the relatively low mitochondrial transfer efficiency during direct co-culture conditions, it appeared unlikely that an MSC-based treatment approach in NDUFS4-related CI deficiency directly enhances cellular energy metabolism. Nevertheless, modulating and mitigating ROS production via MSCs may be an interesting starting point for further in-vivo research studies 
involving established mouse models of mitochondrial NDUFS4 deficiency.

\section{Additional files}

Additional file 1: (A) Western blot analysis from murine NDUFS4 KO and WT cells and from human patient and human control cells. In NDUFS4 KO cells and in NDUFS4-deficient patient cells, no NDUFS4 protein expression is detectable. VDAC (voltage-dependent anion channel) used as loading control. (B) Biochemical measurements from fibroblasts of the NDUFS4-deficient patient, demonstrating severe $\mathrm{Cl}$ deficiency. For details regarding methods that were used to measure OXPHOS enzyme activities in fibroblasts [50]. (TIF $92 \mathrm{~kb}$ )

Additional file 2: (A) Representative fluorescence images showing a mouse fibroblast with LMNB BFP-labelled nucleus, a mouse stem cell (mMSC) with Cox8a GFP-labelled mitochondria and a mouse fibroblast containing mitochondria derived from mMSCs. Cell boundaries are stained with phalloidin. (B) Confocal image depicting a mouse fibroblast with Cox8a RFP-labelled mitochondria in co-culture with an mMSC containing Cox8a GFP-labelled mitochondria. (C) Representative confocal images of human cells. Left: human fibroblasts with Cox8a RFP-labelled mitochondria without transferred mitochondria (upper image) or after mitochondrial transfer via hMSCs (lower image). Right: Cox8a GFP-labelled hMSCs with Cox8a GFP-labelled mitochondria without transferred mitochondria (upper image) or after mitochondrial transfer from human fibroblasts (lower image). Scale bar represents $10 \mu \mathrm{m}$. (TIF $912 \mathrm{~kb}$ )

Additional file 3: (A) Mitochondrial transfer between mouse fibroblasts and mMSCs. Representative fluorescence image of TNTs between fibroblast and mMSC (white arrow). Scale bar represents $10 \mu \mathrm{m}$. (B) Representative flow cytometry analysis images for analysing of mitochondrial transfer. Gating procedure of LMNB RFP positive fibroblasts with transferred Cox8a GFP positive MSC mitochondria. Black arrows indicate sequential analysis steps. Cells (fibroblasts and MSCs) were selected on the basis of cellular size (forward scatter area, FSC-A) and granularity (side scatter area, SSC-A). Only LMNB RFP positive fibroblasts were used for the next step. Cell doublets were excluded by comparing SSC-H (side scatter height) and SSC-W (side scatter width). Double positive fibroblasts were determined. (TIF $670 \mathrm{~kb}$ )

Additional file 4: Is a time-lapse video showing a NDUFS4-deficient mouse fibroblast. Mouse fibroblast mitochondria are labelled (red, Cox8a RFP). The fibroblast contains several green mitochondria (Cox8a GFP labelled) which are derived from mMSCs. Please note the dynamic motility of mitochondria during the time of video recording. (AVI $1038 \mathrm{~kb}$ )

Additional file 5: Is a time-lapse video showing a NDUFS4-deficient human fibroblast. Human fibroblast mitochondria are labelled (red, Cox8a RFP). The fibroblast is in contact with a hMSC cell that appears to transfer green mitochondria (Cox8a GFP labelled). Please note the dynamic motility of mitochondria during the time of video recording. (AVI $1248 \mathrm{~kb}$ )

\section{Abbreviations}

2DG: 2-Deoxy-D-glucose; BN-PAGE: Blue native polyacrylamide ge electrophoresis; BSO: Buthioninesulfoximine; Cl: Complex I; Gal: Galactose; GVHD: Graft-versus-host disease; LHON: Leber hereditary optic neuropathy; MELAS: Mitochondrial encephalomyopathy, lactic acidosis and stroke-like episodes; MERRF: Myoclonic epilepsy with ragged red fibre; MSC: Mesenchymal stem cell; NDUFS4: NADH dehydrogenase ubiquinone Fe-S protein 4; OPA1: Optic atrophy 1 (mitochondrial fusion protein); OXPHOS: Oxidative phosphorylation; ROS: Reactive oxygen species; TNT: Tunnelling nanotube

\section{Acknowledgements}

The authors thank Katharina Raba, Institute for Transplantation Diagnostics and Cell Therapeutics, University Hospital and Medical Faculty of the Heinrich-Heine University Düsseldorf for valuable technical assistance with flow cytometry. Moreover, they thank Professor Arndt Borkhardt, Clinic for Pediatric Oncology, Hematology and Clinical Immunology Düsseldorf, for valuable support and for providing laboratory facilities for our experimental work.

\section{Funding}

This work was supported by a grant from the Forschungskommission of the Medical Faculty of the Heinrich-Heine-University Düsseldorf (\#9772578) and by a grant from the "Elterninitiative Kinderkrebsklinik e.V." (Düsseldorf). FB was supported by a fellowship from the German Research Foundation/ Deutsche Forschungsgemeinschft (BA5758/1-1). ASR was supported by SFB 974 Project B09. FD was supported by a grant from the German Research Foundation/Deutsche Forschungsgemeinschaft (DI 1731/2-1).

\section{Availability of data and materials}

All data generated or analysed during this study are included in this published article (and its supplementary information files).

\section{Authors' contributions}

MM performed the experiments, analysed the data and wrote the manuscript. KD contributed to the study design and performed experiments. AS performed experiments. ÖD provided human and mouse MSCs. FB performed BN-PAGE experiments. AKK assisted in confocal microscopy and interpretation of confocal images. ASR provided OPA1 knockout cells and gave intellectual input. WJHK and PHGMW provided NDUFS4 knockout fibroblasts and gave intellectual input. RJR performed biochemical measurements of patient fibroblasts and provided data about the NDUFS4 mutation. EM provided intellectual input and laboratory resources. RM contributed to the study design and provided intellectual input. FD supervised the work of MM, contributed to the study design, analysed the data and wrote the manuscript. All authors discussed the results and implications and commented on the manuscript at all stages. All authors read and approved the final manuscript.

\section{Competing interests}

The authors declare that they have no competing interests.

\section{Consent for publication}

Not applicable.

\section{Ethics approval and consent to participate}

This study was approved by the Ethic Committee of the Medical Faculty of the Heinrich-Heine-University (study number: 4272). Informed consent was obtained for the use of the human NDUFS4 patient cell line in this study.

\section{Publisher's Note}

Springer Nature remains neutral with regard to jurisdictional claims in published maps and institutional affiliations.

\section{Author details}

${ }^{1}$ Department of General Pediatrics, Neonatology and Pediatric Cardiology, University Children's Hospital, Heinrich Heine University Düsseldorf, Moorenstraße 5, 40225 Düsseldorf, Germany. ${ }^{2}$ Division of Pediatric Stem Cell Therapy, Clinic for Pediatric Oncology, Hematology and Clinical Immunology, Medical Faculty, Heinrich Heine University Medical Center, Düsseldorf, Germany. ${ }^{3}$ Institute of Biochemistry and Molecular Biology I, Medical Faculty, Heinrich Heine University, Düsseldorf, Germany. ${ }^{4}$ Department of Biochemistry (286), Radboud Institute for Molecular Life Sciences, Radboud University Nijmegen (Radboudumc), Nijmegen, The Netherlands. ${ }^{5}$ Radboud Center for Mitochondrial Medicine (RCMM), Department of Pediatrics, 774 Translational Metabolic Laboratory (TML), Radboud University Medical Center, P.O. Box 91016500 HB Nijmegen, The Netherlands.

Received: 21 March 2017 Revised: 17 May 2017

Accepted: 30 May 2017 Published online: 24 June 2017

\section{References}

1. Kruse SE, Watt WC, Marcinek DJ, Kapur RP, Schenkman KA, Palmiter RD. Mice with mitochondrial complex I deficiency develop a fatal encephalomyopathy. Cell Metab. 2008;7(4):312-20.

2. Liu CS, Chang JC, Kuo SJ, Liu KH, Lin TT, Cheng WL, Chuang SF. Delivering healthy mitochondria for the therapy of mitochondrial diseases and beyond. Int J Biochem Cell Biol. 2014;53:141-6.

3. Spees JL, Olson SD, Whitney MJ, Prockop DJ. Mitochondrial transfer between cells can rescue aerobic respiration. Proc Natl Acad Sci U S A. 2006;103(5):1283-8. 
4. Alam MT, Manjeri GR, Rodenburg RJ, Smeitink JA, Notebaart RA, Huynen M, Willems PH, Koopman WJ. Skeletal muscle mitochondria of NDUFS4-/- mice display normal maximal pyruvate oxidation and ATP production. Biochim Biophys Acta. 2015;1847:526-33.

5. Distelmaier F, Valsecchi F, Liemburg-Apers DC, Lebiedzinska M, Rodenburg RJ, Heil S, Keijer J, Fransen J, Imamura H, Danhauser K, et al. Mitochondrial dysfunction in primary human fibroblasts triggers an adaptive cell survival program that requires AMPK-alpha. Biochim Biophys Acta. 2015;1852(3): 529-40.

6. Munnich A, Rustin P. Clinical spectrum and diagnosis of mitochondrial disorders. Am J Med Genet. 2001;106:4-17.

7. Leong DW, Komen JC, Hewitt CA, Arnaud E, McKenzie M, Phipson B, Bahlo M, Laskowski A, Kinkel SA, Davey GM, et al. Proteomic and metabolomic analyses of mitochondrial complex I-deficient mouse model generated by spontaneous B2 short interspersed nuclear element (SINE) insertion into $\mathrm{NADH}$ dehydrogenase (ubiquinone) Fe-S protein 4 (Ndufs4) gene. J Biol Chem. 2012;287(24):20652-63.

8. McKenzie M, Ryan MT. Assembly factors of human mitochondrial complex I and their defects in disease. IUBMB Life. 2010;62(7):497-502.

9. Valsecchi F, Monge C, Forkink M, de Groof AJ, Benard G, Rossignol R, Swarts HG, van Emst-de Vries SE, Rodenburg RJ, Calvaruso MA, et al. Metabolic consequences of NDUFS4 gene deletion in immortalized mouse embryonic fibroblasts. Biochim Biophys Acta. 2012;1817(10):1925-36.

10. Budde SM, van den Heuvel LP, Janssen AJ, Smeets RJ, Buskens CA, DeMeirleir L, Van Coster R, Baethmann M, Voit T, Trijbels JM, et al. Combined enzymatic complex I and III deficiency associated with mutations in the nuclear encoded NDUFS4 gene. Biochem Biophys Res Commun. 2000;275(1):63-8.

11. Leshinsky-Silver E, Lebre AS, Minai L, Saada A, Steffann J, Cohen S, Rotig A, Munnich A, Lev D, Lerman-Sagie T. NDUFS4 mutations cause Leigh syndrome with predominant brainstem involvement. Mol Genet Metab. 2009;97(3):185-9.

12. Petruzzella V, Papa S. Mutations in human nuclear genes encoding for subunits of mitochondrial respiratory complex I: the NDUFS4 gene. Gene. 2002;286:149-54.

13. Friedenstein AJ, Piatetzky-Shapiro II, Petrakova KV. Osteogenesis in transplants of bone marrow cells. J Embryol Exp Morph. 1966;16(3):381-90.

14. Friedenstein AJ, Chailakhjan RK, Lalykina KS. The developement of fibroblast colonies in monolayer cultures of guinea-pig bone marrow and spleen cells. Cell Prolif. 1970;3(4):393-403.

15. Bernardo ME, Fibbe WE. Safety and efficacy of mesenchymal stromal cell therapy in autoimmune disorders. Ann N Y Acad Sci. 2012;1266:107-17.

16. Horwitz EM, Prockop DJ, Fitzpatrick LA, Koo WWK, Gordon PL, Neel M, Sussman M, Orchard P, Marx JC, Pyeritz RE, et al. Transplantability and therapeutic effects of bone marrow-derived mesenchymal cells in children with osteogenesis imperfecta. Nat Med. 1999;5(3):309-13.

17. Jiao H, Shi K, Zhang W, Yang L, Yang L, Guan F, Yang B. Therapeutic potential of human amniotic membrane-derived mesenchymal stem cells in APP transgenic mice. Oncol Lett. 2016;12(3):1877-83.

18. Le Blanc K, Rasmusson I, Sundberg B, Götherström C, Hassan M, Uzunel M, Ringdén $\mathrm{O}$. Treatment of severe acute graft-versus-host disease with third party haploidentical mesenchymal stem cells. Lancet. 2004;363(9419):1439-41.

19. Blazar BR, Murphy WJ, Abedi M. Advances in graft-versus-host disease biology and therapy. Nat Rev Immunol. 2012;12(6):443-58.

20. Krasnodembskaya A, Song Y, Fang X, Gupta N, Serikov V, Lee JW, Matthay MA. Antibacterial effect of human mesenchymal stem cells is mediated in part from secretion of the antimicrobial peptide LL-37. Stem Cells. 2010; 28(12):2229-38.

21. Phinney DG, Di Giuseppe M, Njah J, Sala E, Shiva S, St Croix CM, Stolz DB, Watkins SC, Di YP, Leikauf GD, et al. Mesenchymal stem cells use extracellular vesicles to outsource mitophagy and shuttle microRNAs. Nat Commun. 2015;6:8472.

22. Plotnikov EY, Khryapenkova TG, Vasileva AK, Marey MV, Galkina SI, Isaev NK, Sheval EV, Polyakov VY, Sukhikh GT, Zorov DB. Cell-to-cell cross-talk between mesenchymal stem cells and cardiomyocytes in co-culture. J Cell Mol Med. 2008;12(5A):1622-31.

23. Rustom A, Saffrich R, Markovic I, Walther P, Gerdes H-H. Nanotubular highways for intercellular organelle transport. Science. 2004;303(5660):1007-10.

24. Abounit S, Zurzolo C. Wiring through tunneling nanotubes - from electrical signals to organelle transfer. J Cell Sci. 2012;125(5):1089-98.
25. Pallotti F, Lenaz G. Isolation and subfractionation of mitochondria from animal cells and tissue culture lines. Methods Cell Biol. 2001;65:1-35.

26. Koopman WJ, Distelmaier F, Esseling JJ, Smeitink JA, Willems PH. Computerassisted live cell analysis of mitochondrial membrane potential, morphology and calcium handling. Methods. 2008;46(4):304-11.

27. Calvaruso MA, Smeitink J, Nijtmans L. Electrophoresis techniques to investigate defects in oxidative phosphorylation. Methods. 2008;46(4):281-7.

28. Pasquier J, Guerrouahen BS, Al Thawadi H, Ghiabi P, Maleki M, Abu-Kaoud N, Jacob A, Mirshahi M, Galas L, Rafii S, et al. Preferential transfer of mitochondria from endothelial to cancer cells through tunneling nanotubes modulates chemoresistance. J Transl Med. 2013:11:94.

29. Plotnikov EY, Khryapenkova TG, Galkina SI, Sukhikh GT, Zorov DB. Cytoplasm and organelle transfer between mesenchymal multipotent stromal cells and renal tubular cells in co-culture. Exp Cell Res. 2010;316(15):2447-55.

30. Cho YM, Kim JH, Kim M, Park SJ, Koh SH, Ahn HS, Kang GH, Lee JB, Park KS, Lee HK. Mesenchymal stem cells transfer mitochondria to the cells with virtually no mitochondrial function but not with pathogenic mtDNA mutations. PloS One. 2012;7(3), e32778.

31. Marroquin LD, Hynes J, Dykens JA, Jamieson JD, Will Y. Circumventing the Crabtree effect: replacing media glucose with galactose increases susceptibility of HepG2 cells to mitochondrial toxicants. Toxicol Sci. 2007; 97(2):539-47.

32. Li L, Fath MA, Scarbrough PM, Watson WH, Spitz DR. Combined inhibition of glycolysis, the pentose cycle, and thioredoxin metabolism selectively increases cytotoxicity and oxidative stress in human breast and prostate cancer. Redox Biol. 2015;4:127-35.

33. Wick AN, Drury DR, Nakada HI, Wolfe JB. Localisation of the primary metabolic block produced by 2-deoxyglucose. J Biol Chem. 1956;224(2):963-9.

34. Ranzinger J, Rustom A, Abel M, Leyh J, Kihm L, Witkowski M, Scheurich P, Zeier M, Schwenger V. Nanotube action between human mesothelial cells reveals novel aspects of inflammatory responses. PloS One. 2011;6(12), e29537.

35. Chen L, Gong Q, Stice JP, Knowlton AA. Mitochondrial OPA1, apoptosis, and heart failure. Cardiovasc Res. 2009;84(1):91-9.

36. Holzerova E, Prokisch H. Mitochondria: Much ado about nothing? How dangerous is reactive oxygen species production? Int J Biochem Cell Biol. 2015;63:16-20.

37. Distelmaier F, Koopman WJ, van den Heuvel LP, Rodenburg RJ, Mayatepek E, Willems PH, Smeitink JA. Mitochondrial complex I deficiency: from organelle dysfunction to clinical disease. Brain. 2009;132(Pt 4):833-42.

38. Assouline Z, Jambou M, Rio M, Bole-Feysot C, de Lonlay P, Barnerias C, Desquerre I, Bonnemains C, Guillermet C, Steffann J, et al. A constant and similar assembly defect of mitochondrial respiratory chain complex I allows rapid identification of NDUFS4 mutations in patients with Leigh syndrome. Biochim Biophys Acta. 2012;1822(6):1062-9.

39. Gerdes HH, Bukoreshtliev NV, Barroso JF. Tunneling nanotubes: a new route for the exchange of components between animal cells. FEBS Lett. 2007; 581(11):2194-201.

40. Zhu D, Tan KS, Zhang X, Sun AY, Sun GY, Lee JCM. Hydrogen peroxide alters membrane and cytoskeleton properties and increases intercellular connections in astrocytes. J Cell Sci. 2005;118(16):3695-703.

41. Liu K, Ji K, Guo L, Wu W, Lu H, Shan P, Yan C. Mesenchymal stem cells rescue injured endothelial cells in an in vitro ischemia-reperfusion model via tunneling nanotube like structure-mediated mitochondrial transfer. Microvasc Res. 2014:92:10-8.

42. Ahmad T, Mukherjee S, Pattnaik B, Kumar M, Singh S, Kumar M, Rehman R, Tiwari BK, Jha KA, Barhanpurkar AP, et al. Miro1 regulates intercellular mitochondrial transport \& enhances mesenchymal stem cell rescue efficacy. EMBO J. 2014;33(9):994-1010.

43. Le Blanc K, Davies LC. Mesenchymal stromal cells and the innate immune response. Immunol Lett. 2015;168(2):140-6.

44. Jiang D, Gao F, Zhang Y, Wong DS, Li Q, Tse HF, Xu G, Yu Z, Lian Q. Mitochondrial transfer of mesenchymal stem cells effectively protects corneal epithelial cells from mitochondrial damage. Cell Death Dis. 2016; 7(11), e2467.

45. Youle RJ, van der Bliek AM. Mitochondrial fission, fusion, and stress. Science. 2012;337(6098):1062-5.

46. Valsecchi F, Grefte S, Roestenberg P, Joosten-Wagenaars J, Smeitink JA, Willems PH, Koopman WJ. Primary fibroblasts of NDUFS4(-/-) mice display increased ROS levels and aberrant mitochondrial morphology. Mitochondrion. 2013;13(5):436-43. 
47. Kim Y, Jo SH, Kim WH, Kweon OK. Antioxidant and anti-inflammatory effects of intravenously injected adipose derived mesenchymal stem cells in dogs with acute spinal cord injury. Stem Cell Res Ther. 2015;6:229.

48. Valle-Prieto A, Conget PA. Human mesenchymal stem cells efficiently manage oxidative stress. Stem Cells Dev. 2010;19(12):1885-93.

49. Ayatollahi M, Hesami Z, Jamshidzadeh A, Gramizadeh B. Antioxidant effects of bone marrow mesenchymal stem cell agains carbon tetrachlorideinduced oxidative damage in rat livers. Int J Org Transplant Med. 2014;5(4): 166-73.

50. Rodenburg RJ. Biochemical diagnosis of mitochondrial disorders. J Inherit Metab Dis. 2011; 34(2):283-92.

Submit your next manuscript to BioMed Central and we will help you at every step:

- We accept pre-submission inquiries

- Our selector tool helps you to find the most relevant journal

- We provide round the clock customer support

- Convenient online submission

- Thorough peer review

- Inclusion in PubMed and all major indexing services

- Maximum visibility for your research

Submit your manuscript at www.biomedcentral.com/submit
Biomed Central 